\title{
Measuring Inner Layer Capacitance with the Colloidal Probe Technique
}

\author{
Alexander M. Smith ${ }^{\circledR}$, Plinio Maroni, Michal Borkovec and Gregor Trefalt *®i] \\ Department of Inorganic and Analytical Chemistry, University of Geneva, Sciences II, 30 Quai Ernest-Ansermet, \\ 1205 Geneva, Switzerland; Alexander.Smith@unige.ch (A.M.S.); plinio.maroni@unige.ch (P.M.); \\ michal.borkovec@unige.ch (M.B.) \\ * Correspondence: gregor.trefalt@unige.ch
}

Received: 26 October 2018; Accepted: 22 November 2018; Published: 27 November 2018

\begin{abstract}
The colloidal probe technique was used to measure the inner layer capacitance of an electrical double layer. In particular, the forces were measured between silica surfaces and sulfate latex surfaces in solutions of monovalent salts of different alkali metals. The force profiles were interpreted with Poisson-Boltzmann theory with charge regulation, whereby the diffuse layer potential and the regulation properties of the interface were obtained. While the diffuse layer potential was measured in this fashion in the past, we are able to extract the regulation properties of the inner layer, in particular, its capacitance. We find systematic trends with the type of alkali metal ion and the salt concentration. The observed trends could be caused by difference in ion hydration, variation of the binding capacitance, and changes of the effective dielectric constant within the Stern layer. Our results are in agreement with recent experiments involving the water-silica interface based on a completely independent method using $X$-ray photoelectron spectroscopy in a liquid microjet. This agreement confirms the validity of our approach, which further provides a means to probe other types of interfaces than silica.
\end{abstract}

Keywords: surface forces; DLVO; charge regulation; inner layer capacitance

\section{Introduction}

A solid surface immersed in an aqueous solution usually acquires charge at the solid-liquid interface [1,2]. This process is governed by adsorption of ions and/or ionization of surface groups within a thin and compact layer, which is situated close to the interface. This charge is then neutralized by an accumulation of counterions from the electrolyte solution in a so-called diffuse layer, which may gradually extend far away from the interface. In this fashion, an electrical double layer is formed and its properties are relevant for many phenomena, for example, stabilization of colloidal particle suspensions, electrophoretic mobility of charged particles, or the formation colloidal crystals [1,2]. Such phenomena are important in many practical applications, such as waste water treatment, paper making, particle fabrication, and microfluidics [3-6].

The diffuse part of the double layer is rather well understood, and can be accurately described with the Poisson-Boltzmann theory, especially for monovalent electrolytes $[1,2,7,8]$. However, less is known about the compact, inner layer, the properties of which are determined by ionization of the surface groups, specific ion adsorption, and interfacial water structure. Already a long time ago, Stern [9] has argued that ions can approach an interface only to a finite distance. This effect leads to an additional capacitance, which influences the charging process of the inner layer. The charging of the inner layer is further influenced by specific adsorption of protons or other ions, and the overall layer can be characterized by an inner capacitance. While such capacitances can be routinely measured with electrochemical techniques for conducting planar substrates [7], obtaining similar 
information for non-conducting substrates, and especially for colloidal particles, remains a challenge. One possible approach is to study ion adsorption and dissociation of ionizable surface groups in particle suspensions [10-13] and to extract the Stern capacitance by fitting the measured adsorption isotherms with suitable adsorption models $[10,14]$. Another approaches are second harmonic generation and X-ray reflectivity techniques, which provide additional information about the water structure near the water-solid interface, and also permit to extract the inner capacitance in some situations [15-18]. Direct measurements of the inner capacitance in such systems remain difficult, however. This aim was only recently achieved by studying a silica particle suspension with X-ray photoelectron spectroscopy (XPS) in a microjet [14,19]. With this technique the surface potential was measured, and by combining this information with results obtained from titration and electrokinetic measurements, it was possible to extract the Stern layer capacitance. Given the complexity of this approach, however, alternative techniques capable to measure the capacitance of the inner layer in such systems more directly would certainly be welcome.

The aim of the present article is to demonstrate that direct force measurements hold substantial promise in this respect. Such experiments may rely on the surface force apparatus, colloidal probe technique, or optical tweezers techniques [20-24]. These measurements yield forces between charged interfaces and provide access to van der Waals and double layer forces acting between these surfaces, and can be interpreted with the theory of Derjaguin, Landau, Verwey, and Overbeek (DLVO) $[1,2,25,26]$. Here, we mainly focus on double layer forces, which become dominant at lower salt levels, and can be quantified by the Poisson-Boltzmann (PB) theory $[1,2,27,28]$. This theory permits to extract the diffuse layer potential from the force profiles at larger distances, and such measurements were reported by several studies $[22,23,29,30]$.

At shorter distances, however, the boundary conditions used in the PB theory become important. Many studies rely on the constant charge (CC) or constant potential (CP) conditions [1,2]. These conditions stipulate that the surface charge or the diffuse layer potential remain constant upon approach. Mostly, however, neither of these conditions is applicable. In reality, the surface charge as well as the diffuse layer potential regulate upon approach [31-35]. Such charge regulation effects can be included into the PB description, whereby the simplest approach is the constant regulation (CR) model $[32,33,35]$. This model introduces one additional regulation parameter, $p$, which characterizes the regulation properties of the interface. While this model recovers the classical CC conditions with $p=1$ and the CP conditions with $p=0$, this parameter can assume any value $p \leq 1$, but normally remains positive $[33,35,36]$. The simplification introduced by the CR model is that this parameter remains constant upon approach. While this constancy is not exact, measured force profiles and model calculations reveal that the CR approximation is mostly highly accurate [33,37]. Moreover, the regulation parameter defined in this fashion reflects the properties of the isolated interface, which should simplify its interpretation.

However, the values of these parameters were so far not interpreted any further, in spite of the fact that the regulation parameter was extracted from direct force measurements in some situations [22,37]. Here we will demonstrate that from this parameter one can calculate the capacitance of the inner layer. We exemplify the feasibility of this approach by direct force measurements between pairs of silica and sulfate latex particles with the colloidal probe technique. Moreover, our results for the water-silica interface agree reasonably well with recent measurements of the Stern capacitance with X-ray photoelectron spectroscopy by Brown et al. [14,19]. This agreement with a completely independent technique provides further support of the validity of the present approach. 


\section{Experimental Setup and Methods}

\subsection{Materials}

Electrolyte solutions of $\mathrm{LiCl}, \mathrm{NaCl}, \mathrm{KCl}$ and $\mathrm{CsCl}$ (Sigma-Aldrich, $\geq 99 \%$ ) were prepared using ultrapure water and adjusted to either $\mathrm{pH} 4.0$ with hydrochloric acid or to $\mathrm{pH} 10.0$ with the corresponding alkali metal hydroxide.

Silica particles of average diameter $5.2 \mu \mathrm{m}$ were purchased from Bangs Laboratories Inc., USA. Particles were attached to tipless cantilevers (MicroMash) using epoxy glue, and also sprinkled over quartz substrates. Before measurements, the particles were heat-treated at $1150{ }^{\circ} \mathrm{C}$ for $3 \mathrm{~h}$. This sintering process serves not only to firmly attach the particles to substrates and cantilevers, but also shrinks the particles to $4.4 \mu \mathrm{m}$, reduces the surface roughness, and removes any traces of glue [38]. Immediately prior to force measurements, silica substrates and cantilevers were rinsed thoroughly with ethanol and ultrapure water, then dried and cleaned in air plasma for $20 \mathrm{~min}$.

Stock suspensions of polystyrene latex particles functionalized with sulfate groups (Invitrogen Corp., $2.9 \mu \mathrm{m}$ ) were dialyzed with a cellulose ester membrane against ultrapure water until the conductivity was below $70 \mu \mathrm{S} \mathrm{m}^{-1}$. Prior to the force measurements, a substrate was cleaned in piranha solution and then functionalized with 3-(ethoxydimethylsilyl)propylamine via vapor deposition. The substrate was mounted in the fluid cell, rinsed thoroughly with ultrapure water, and the particle suspension was introduced. After enough particles were attached to the positively charged substrate, the remaining unattached particles were removed by flushing the cell with the electrolyte in question. A similarly functionalized tip-less cantilever was then used to press and pick up particles under water to avoid exposing the hydrophobic particles to air.

\subsection{Direct Force Measurements}

A closed-loop AFM (MFP-3D, Asylum Research) mounted on an inverted optical microscope (Olympus IX 73) was used to measure forces between pairs of similar colloidal particles. The particle on the cantilever was aligned above a substrate particle to within $50 \mathrm{~nm}$ using the horizontal scanner of the AFM. After centering a pair of particles, about 200 force-distance curves were measured at velocity $300 \mathrm{~nm} \mathrm{~s}^{-1}$. The deflection signal was converted to force-distance profiles by subtracting the baseline and by fitting the constant compliance region. The spring constant of the cantilever was measured using the method developed by Sader et al. [39], which relies on lateral dimensions of the cantilever and its frequency response. The respective spring constants were typically between 0.2 and $0.5 \mathrm{~N} \mathrm{~m}^{-1}$. Forces obtained from repeated approach curves were averaged, typically leading to a force resolution of about $2 \mathrm{pN}$ and to distances of about $0.3 \mathrm{~nm}$. The averaged force curves were finally normalized by the effective radius $R_{\text {eff }}$, which is calculated as $R_{\text {eff }}=R / 2$ for two spheres of equal radius $R$.

\subsection{DLVO Theory}

Forces are analyzed using the DLVO theory, which surmises that the total interaction is a sum of van der Waals force $F_{\mathrm{vdW}}$ and double layer force $F_{\mathrm{dl}}$, namely $[1,2]$ :

$$
F=F_{\mathrm{vdW}}+F_{\mathrm{dl}}
$$

The van der Waals force is calculated with the non-retarded expression within the Derjaguin approximation [1]:

$$
\frac{F_{\mathrm{vdW}}}{R_{\mathrm{eff}}}=-\frac{H}{6 h^{2}}
$$

where $H$ is the Hamaker constant, $h$ is the separation distance. 
For calculation of double layer forces the PB equation for 1:1 electrolyte in the plate-plate geometry at a separation distance $h$ is solved numerically [1]:

$$
\frac{d^{2} \psi(x)}{d x^{2}}=\frac{\kappa^{2}}{\beta q} \sinh (\beta q \psi)
$$

where $\psi(x)$ is the electrical potential profile, $q$ is the elementary charge, $\beta=1 /\left(k_{\mathrm{B}} T\right)$ is the inverse thermal energy with $k_{\mathrm{B}}$ being the Boltzmann constant and $T$ absolute temperature, and $\kappa$ is the inverse Debye screening length defined as:

$$
\kappa=\sqrt{\frac{2 \beta q^{2} c}{\varepsilon \varepsilon_{0}}}
$$

where $c$ is the monovalent electrolyte concentration, $\varepsilon_{0}$ is the vacuum permittivity, and $\varepsilon$ is the relative dielectric permittivity. For the latter quantity $\varepsilon=80$ is used as appropriate for water at $25{ }^{\circ} \mathrm{C}$. Symmetry of the problem permits to solve Equation (3) in a half-space $x \in[0, h / 2]$, the full geometry is then defined by the two identical plates situated at $x= \pm h / 2$. The essential part of our approach is to use the CR boundary condition [33]:

$$
\left.\varepsilon \varepsilon_{0} \frac{d \psi}{d x}\right|_{x=h / 2}=\sigma-C_{\text {in }}\left[\psi(h / 2)-\psi_{\mathrm{dl}}\right]
$$

where $\sigma$ is the surface charge density of the isolated interface, $\psi_{\mathrm{dl}}$ is the diffuse layer potential of the isolated interface, and $C_{\text {in }}$ is the inner layer capacitance of the isolated interface. Here we refer to surface charge density $\sigma$ as the charge which is compensating the diffuse layer charge. Note that isolated interfaces have identical properties as interfaces at infinite separation. Symmetry of the problem implies the second boundary condition:

$$
\left.\frac{d \psi}{d x}\right|_{x=0}=0
$$

The surface charge density and diffuse layer potential of the isolated interface are related by the Grahame relation [1]:

$$
\sigma=\frac{2 \varepsilon \varepsilon_{0} \kappa}{\beta q} \sinh \left(\frac{\beta q \psi_{\mathrm{dl}}}{2}\right)
$$

The inner layer capacitance can be also represented with a regulation parameter [33]:

$$
p=\frac{C_{\mathrm{dl}}}{C_{\mathrm{dl}}+C_{\mathrm{in}}}
$$

where $C_{\mathrm{dl}}$ is the diffuse layer capacitance given by [1]:

$$
C_{\mathrm{dl}}=\varepsilon \varepsilon_{0} \kappa \cosh \left(\frac{\beta q \psi_{\mathrm{dl}}}{2}\right)
$$

Both diffuse layer potential and inner layer capacitance enter the regulation parameter which represents a description of an isolated surface. The regulation parameter takes simple values for constant potential (CP) boundary conditions $p=0$ and constant charge boundary (CC) conditions $p=1$.

When the electrical potential profile is known for a separation distance the disjoining pressure can be obtained as [1]:

$$
\Pi(h)=\frac{2 c}{\beta}\left[\cosh \left(\beta q \psi_{\mathrm{M}}\right)-1\right]
$$


where $\psi_{\mathrm{M}}=\psi(0)$ is the electrical potential at the mid-plane. The force is obtained by the integration of the pressure profile and by using the Derjaguin approximation [1]:

$$
\frac{F_{\mathrm{dl}}}{R_{\mathrm{eff}}}=2 \pi \int_{h}^{\infty} \Pi\left(h^{\prime}\right) d h^{\prime}
$$

When the experimental force profiles are fitted, the salt concentration is fixed to the nominal value and the Hamaker constant is fixed to the value obtained at concentrations above $300 \mathrm{mM}$. The only two fitting parameters left are the diffuse layer potential $\psi_{\mathrm{dl}}$ and the regulation parameter $p$. These two quantities are extracted by fitting the DLVO force profile to the experimental data.

\subsection{Basic Stern Model}

The basic Stern model was successfully used to model protentiometric and electrokinetic data for silica surfaces $[10,12,33]$, and it will also be used to the interpretation of the present data here. The relevant notions of the model are summarized here.

The model considers a surface ionization reaction of the silanol group:

$$
\mathrm{Si}-\mathrm{OH} \rightleftarrows \mathrm{Si}-\mathrm{O}^{-}+\mathrm{H}^{+}
$$

The respective surface site densities $\Gamma_{\mathrm{SiOH}}$ and $\Gamma_{\mathrm{SiO}^{-}}$are related by the mass action law:

$$
\frac{a_{\mathrm{H}} \Gamma_{\mathrm{SiO}^{-}}}{\Gamma_{\mathrm{SiOH}}}=K \exp \left(q \beta \psi_{0}\right)
$$

whereby $a_{\mathrm{H}}$ is the bulk proton activity, $K$ the intrinsic ionization constant, and $\psi_{0}$ the electric surface potential in the plane of the surface. One commonly also uses the abbreviations $\mathrm{pH}=-\log a_{\mathrm{H}}$ and $\mathrm{pK}=-\log K$. The total site density is given by:

$$
\Gamma_{0}=\Gamma_{\mathrm{SiOH}}+\Gamma_{\mathrm{SiO}^{-}}
$$

Equations (13) and (14) can be conveniently combined as:

$$
\sigma=\frac{q \Gamma_{0}}{2}\left[\tanh \left(\frac{\beta q \psi_{N}}{2}-\frac{\beta q \psi_{0}}{2}\right)-1\right]
$$

where $\psi_{\mathrm{N}}=(\mathrm{pK}-\mathrm{pH}) \ln 10 /(\beta q)$ is the Nernst potential. The diffuse layer potential $\psi_{\mathrm{dl}}$ in the plane of the origin of the diffuse layer introduced above is assumed to be related to the surface potential $\psi_{0}$ though the Stern capacitance [9]:

$$
C_{\mathrm{S}}=\frac{\sigma}{\psi_{0}-\psi_{\mathrm{dl}}}
$$

whereby the surface charge density is given by $\sigma=-q \Gamma_{\mathrm{SiO}^{-}}$. The surface charges density can be found by combining Equations (15) and (16) with the Grahame relation given by Equation (7). The inner capacitance defined in Equation (5) can be obtained from the basic Stern model as

$$
C_{\mathrm{in}}=-\frac{\partial \sigma}{\partial \psi_{\mathrm{dl}}}
$$

Evaluating this derivative, one finds that inner layer capacitance can be considered as a two capacitors in series, whereby one contribution stems from the proton binding equilibrium and the second is the Stern capacitance [33]:

$$
\frac{1}{C_{\mathrm{in}}}=\frac{1}{C_{\mathrm{bi}}}+\frac{1}{C_{\mathrm{S}}}
$$


where we refer to $C_{b i}$ as the binding capacitance. The latter parameter is given by:

$$
C_{\mathrm{bi}}=\frac{\beta q^{2} \Gamma_{0}}{4 \cosh ^{2}\left(\frac{\beta q \psi_{0}}{2}-\frac{\beta q \psi_{N}}{2}\right)}
$$

Figure 1 presents results of model calculations for the water-silica interface with the basic Stern model, whereby the diffuse layer potentials and various capacitances are presented as a function of salt concentration. The parameters used are taken from literature. In the left column of Figure 1, we use the parameters $\mathrm{pK}=7.5, \Gamma_{0}=8 \mathrm{~nm}^{-2}$, and $C_{\mathrm{S}}=2.9 \mathrm{~F} / \mathrm{m}^{2}$, which were extracted from potentiometric titrations by Hiemstra et al. [10]. In the right column of the same figure we use a lower value for the Stern layer capacitance $C_{S}=0.5 \mathrm{~F} / \mathrm{m}^{2}$ in accordance with the recently measured values from Brown et al. [14]. The other parameters are kept the same. The basic Stern model predicts the increase of diffuse layer potential with increasing salt concentration. The model further predicts an increase of the binding capacitance and consequently also of the inner capacitance, see Figure $1 \mathrm{~b}$. However, since the inner capacitance is mainly determined by the lower of the two values according to Equation (18), the inner capacitance is a good approximation for the Stern capacitance at high salt concentration. For higher value of Stern capacitance the inner capacitance increase with concentration is stronger, since the contribution of the binding capacitance is more pronounced.
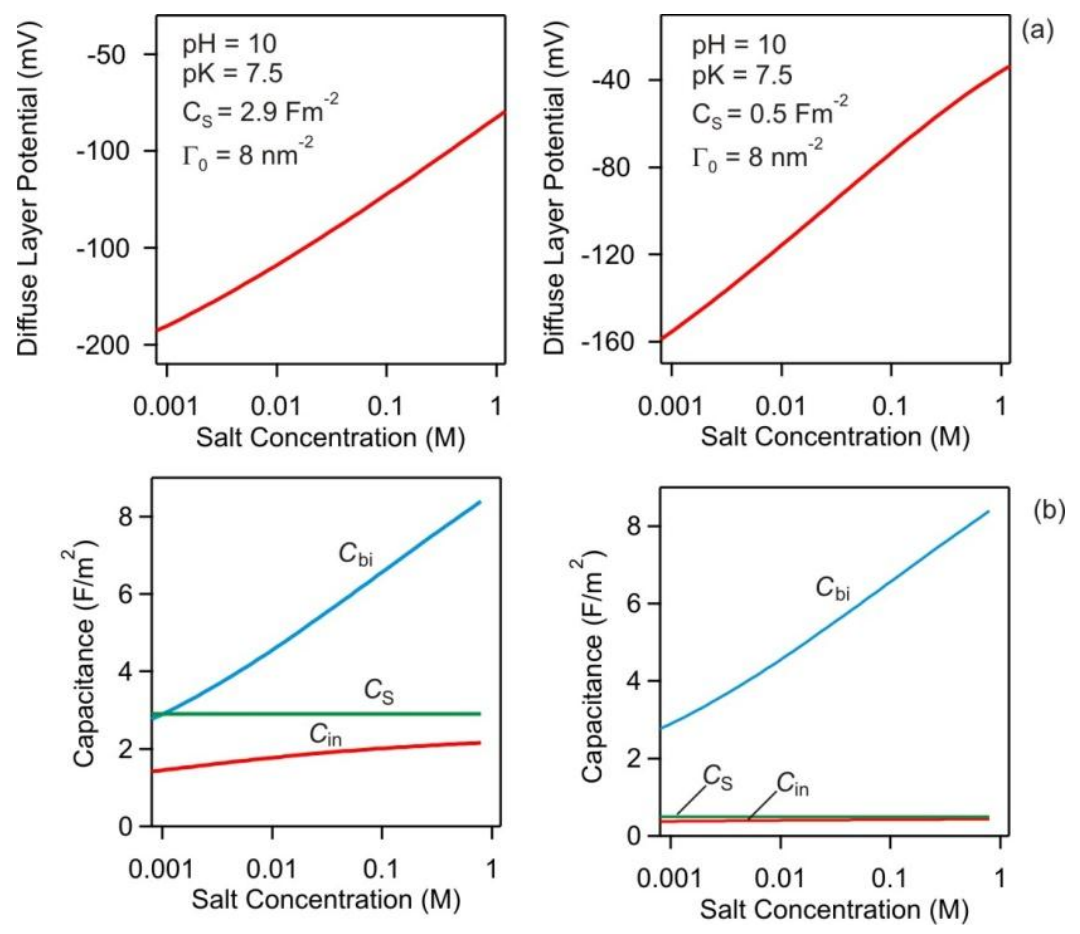

Figure 1. Predictions from the basic Stern model for (a) diffuse layer potential and (b) capacitances versus monovalent salt concentration. The parameters $\mathrm{pK}=7.5, \Gamma_{0}=8 \mathrm{~nm}^{-2}$, and $C_{\mathrm{S}}=2.9 \mathrm{~F} / \mathrm{m}^{2}$ proposed by Hiemstra et al. [10], are used in the left column and in the right column the Stern capacitance is reduced to $C_{S}=0.5 \mathrm{~F} / \mathrm{m}^{2}$ while other parameters are kept the same [14].

\section{Results and Discussion}

This article presents measurements of the Stern capacitance in monovalent electrolytes by direct force measurements based on the atomic force microscope (AFM). Forces between pairs of silica and sulfate latex particles are measured and interpreted with the PB theory with constant regulation (CR) boundary conditions. Based on this analysis, the inner capacitance can be extracted. This capacitance shows systematic trends with the electrolyte concentration and the type of ion. 


\subsection{Quantification of Measured Forces Profiles by DLVO Theory}

Figure 2 compares typical normalized force profiles measured in solutions of $\mathrm{KCl}$ at $\mathrm{pH} 4$ for silica and sulfate latex particles. At lower salt concentrations, the forces are repulsive and follow an exponential law as evident from the linear dependence in the semi-logarithmic representation shown in Figure 2a. The range of these forces decreases with increasing salt concentration. This behavior is typical for double layer forces [40-42]. At shorter distances, typically below 1-2 nm, the forces between silica particles become strongly repulsive, while the ones between latex particles are attractive and induce a mechanical instability of the cantilever. These short range forces are due to hydration or hydrophobic interactions [38,43-48]. At higher concentrations, the forces are attractive and they are independent of the salt concentration, see Figure $2 b$. This situation is characteristic for van der Waals forces.
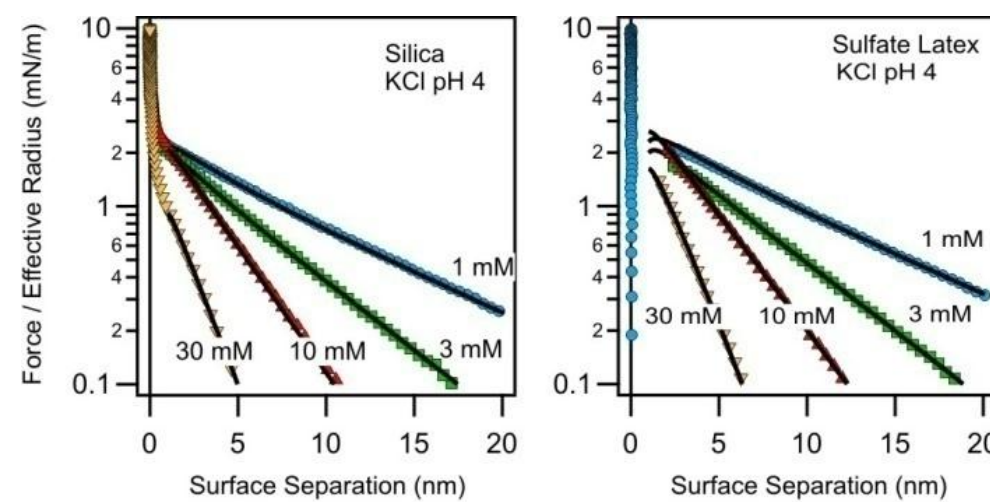

(a)
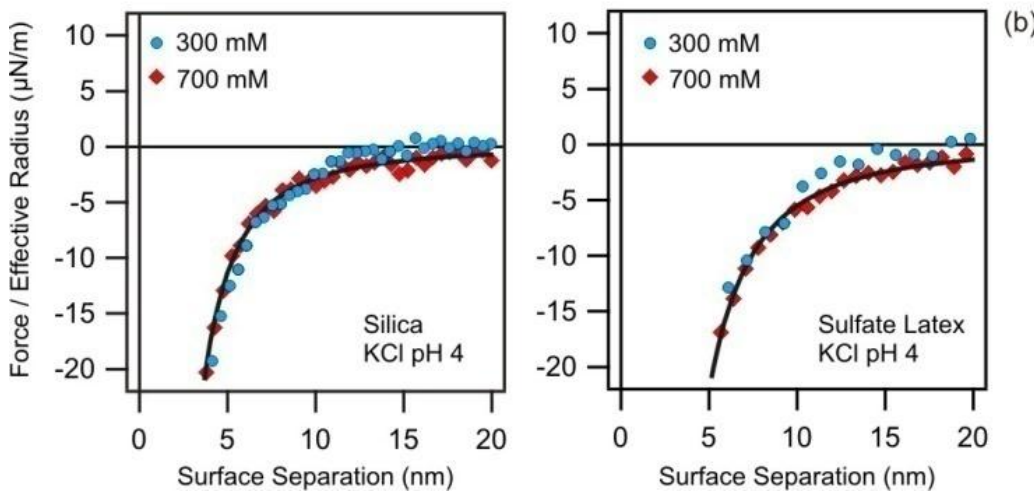

Figure 2. Normalized force profiles measured between silica particles (left column) and sulfate latex particles (right column) in $\mathrm{KCl}$ solutions at $\mathrm{pH}$ 4. DLVO theory fits with constant regulation (CR) approximation are shown as solid lines. (a) Semi-logarithmic representation of the repulsive forces at low salt concentrations, and (b) linear representation of the attractive forces at high salt concentrations.

The force profiles were quantitatively interpreted with DLVO theory. The attractive force profiles observed at high salt concentrations were first fitted to Equation (2) and in this fashion the Hamaker constants were extracted. We found $(1.7 \pm 0.2) \times 10^{-21} \mathrm{~J}$ and $(3.3 \pm 0.3) \times 10^{-21} \mathrm{~J}$ for silica and latex particles, respectively. The values of the Hamaker constants reported above are in good agreement with the values measured earlier for similar particles [30,48,49]. Moreover, the measured Hamaker constant for silica is close to the theoretical value of $1.6 \times 10^{-21} \mathrm{~J}$, which was calculated using accurate dielectric spectra over a wide frequency range [50]. The Hamaker constant determined for latex particles is somewhat lower when compared to the value calculated by the Lifshitz theory for smooth surfaces of $9.0 \times 10^{-21} \mathrm{~J}$ [51]. This discrepancy is probably related to the surface roughness of latex particles [52]. Root mean square (RMS) roughness measured for similarly prepared sulfate latex and silica probes was reported to be $0.8 \mathrm{~nm}$ and $0.7 \mathrm{~nm}$, respectively [48,49]. In the case of silica for the values of RMS 
roughness of just below $1 \mathrm{~nm}$ the Hamaker constant already saturates close to the theoretical value for smooth surfaces [49], while in the case of polystyrene latex particles the discrepancy between the theoretical and roughness-corrected Hamaker constants is still prominent for RMS roughness of about $1 \mathrm{~nm}$ [52]. This difference between the two surfaces is probably related to the different values of Hamaker constants for smooth surfaces.

To interpret the force profiles at lower salt concentrations, the Hamaker constants are fixed to the measured values reported above, and the profiles are fitted to DLVO theory given in Equation (1). Thereby, the double layer forces were calculated by solving the full PB equation with CR boundary conditions numerically. The salt concentrations were fixed to the nominal values and, thus, the fits involve two unknown parameters, namely the diffuse layer potential $\psi_{\mathrm{dl}}$ and the regulation parameter $p$. When the salt concentration was fitted as well, the resulting values would lie within few percent of the nominal values. In the present system, PB theory is expected be accurate, as effects of ion-ion correlations are typically negligible in monovalent electrolytes $[27,28]$.

The resulting fits are shown in Figure 2a as solid lines. One observes that the DLVO theory is capable to describe the measured force profiles in a satisfactory fashion. Marked deviations occur only at distances below $2 \mathrm{~nm}$, where short ranged hydration and hydrophobic forces become important [38,43-48]. These forces are not included in DLVO theory, and for this reason this region was excluded from the fitting process.

Figure 3 provides more details concerning effects of the charge regulation for both types of surfaces, and exemplifies effects of $\mathrm{pH}$ and the type of cation. Experimental force profiles are compared with the best fit involving the CR boundary condition, whereby the regulation parameter $p$ is determined. This result is compared with the corresponding CC $(p=1)$ and CP $(p=0)$ cases.
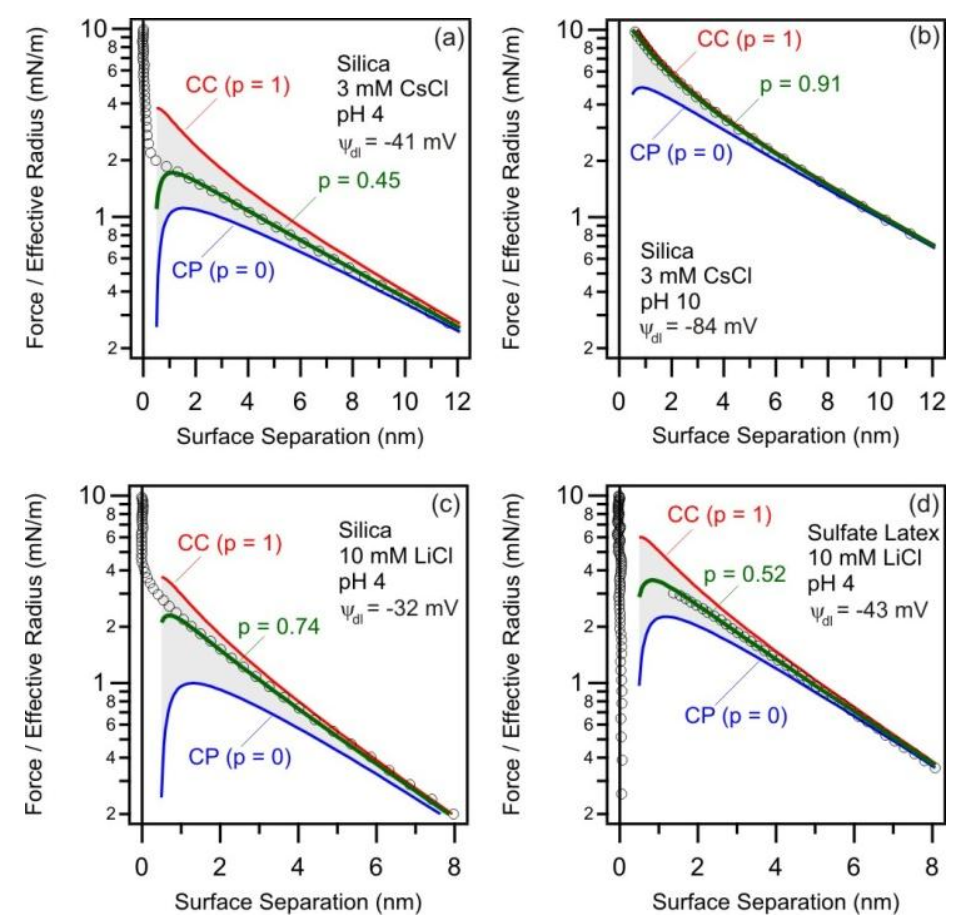

Figure 3. Normalized force profiles measured between (a) silica particles in $3 \mathrm{mM} \mathrm{CsCl}$ solution at $\mathrm{pH} 4$, (b) silica particles in $3 \mathrm{mM} \mathrm{CsCl}$ solution at $\mathrm{pH} 10$, (c) silica particles in $10 \mathrm{mM} \mathrm{LiCl}$ solution at $\mathrm{pH} 4$, and (d) sulfate latex particles in $10 \mathrm{mM}$ at pH 4. DLVO theory fits with constant regulation (CR) approximation are shown with full lines, whereby the values for the best fit for the diffuse layer potential $\psi_{\mathrm{dl}}$ and the regulation parameter are indicated in the figures. The results for constant charge $(\mathrm{CC}, p=1)$ and constant potential $(\mathrm{CP}, p=0)$ are also shown. The region between $\mathrm{CP}$ and $\mathrm{CC}$ is shaded. 
The first row of Figure 3, compares the results for silica particles in $3 \mathrm{mM} \mathrm{CsCl}$ solution at $\mathrm{pH} 4$ (left) and $\mathrm{pH} 10$ (right). The forces at $\mathrm{pH} 4$ are less repulsive than at $\mathrm{pH} 10$, which is reflected by the higher magnitude of the diffuse layer potential $\psi_{\mathrm{dl}}$ for the latter conditions. The respective values for $\psi_{\mathrm{dl}}$ of $-41 \mathrm{mV}$ at $\mathrm{pH} 4$ and $-84 \mathrm{mV}$ at $\mathrm{pH} 10$. The decrease of the potential is due to the fact that more silanol groups are ionized in basic conditions.

More importantly, however, clear differences in the regulation behavior can be pinpointed as well. At $\mathrm{pH} 4$, the surfaces regulate their charge quite easily as the force profile falls roughly in the middle between the $\mathrm{CC}$ and $\mathrm{CP}$ case. At $\mathrm{pH} 10$, on the other hand, the surfaces hardly regulate their charge, and the measured force profile almost follows the $\mathrm{CC}$ case. This behavior can be quantified by the respective values of the regulation parameters. The surfaces regulate easily at $\mathrm{pH} 4$ and one has $p=0.45$, while the hardly regulating surfaces at $\mathrm{pH} 10$ are characterized by $p=0.91$. Thus, with increasing $\mathrm{pH}$, the extent of charge regulation of the silica surface decreases.

Marked effects in charge regulation can be also observed with the type of cation. This effect is illustrated in the left column of Figure 3. The force profile measured in $\mathrm{LiCl}$ is markedly shifted toward the $\mathrm{CC}$ profile, while the respective profile in $\mathrm{CsCl}$ lies in between the $\mathrm{CP}$ and $\mathrm{CC}$ situations. Again, this behavior is reflected in the values of regulation parameters. For $\mathrm{LiCl}$ one finds $p=0.74$, while in $\mathrm{CsCl}$ one has $p=0.45$. Thus, the surfaces regulate more easily in $\mathrm{CsCl}$ than in $\mathrm{LiCl}$ solutions.

Going from silica surface to sulfate latex surface in $10 \mathrm{mM} \mathrm{LiCl}$ the regulation parameter reduces from $p=0.74$ to $p=0.52$, while the diffuse layer potential slightly increases as evident from Figure $3 \mathrm{c}$, d. Therefore, the regulation of the sulfate surface is slightly more pronounced as compared to the silica surface.

One should further note that the sign of the diffuse layer potential cannot be determined from the force profiles in the symmetric geometry. However, the negative sign can be easily inferred from the chemical nature of the surface groups. At the conditions investigated, the silanol groups on the silica surface are partially dissociated, and the sulfate groups on the latex surface fully. The dissociated groups are negatively charged in both cases and therefore the diffuse layer potential is also negative, see Equation (7). The fact that these surfaces are negatively charged is further confirmed by electrokinetic measurements $[12,29,53]$.

A comment is in order why the force profiles presented in Figure 2a appear close to linear in the semi-log scale, while the non-linear PB theory is being used. For the conditions shown in Figure $2 \mathrm{a}$, the regulation parameters are close to 0.5 , and the interplay between the non-linearity of the PB equation and charge regulation effects lead to an almost exponential dependence down to contact. In contrast, Figure $3 \mathrm{~b}$ clearly shows the non-exponential dependence at separation distances below $10 \mathrm{~nm}$, here the regulation parameter is close to unity. From the data shown in Figure 2a one might thus naively conclude that Equation (3) could be linearized and the Debye-Hückel approximation could be used to interpret the data. However, to reliably extract diffuse layer potentials and regulation parameters the non-linear PB equation has to be used.

\subsection{Charging and Regulation Behavior of Isolated Surfaces}

Fitting of experimental force profiles with DLVO theory enables one to extract the diffuse layer potential $\psi_{\mathrm{dl}}$ and regulation parameter $p$. These two parameters describe the properties of the isolated surfaces. The diffuse layer potential is related to the surface charge density through the Grahame relation given in Equation (7). The regulation parameter is a measure of the extent of charge regulation upon approach, whereby values close to $p=1$ (CC) reflect hardly any charge regulation, while the smaller the value, the larger the extent of charge regulation becomes.

These two parameters were extracted for different salt concentrations and they are summarized in Figure 4. The columns represent the three systems were investigated, namely forces between silica particles at $\mathrm{pH} 4$, forces between sulfate latex particles at $\mathrm{pH} 4$, and forces between silica particles at $\mathrm{pH}$ 10. These three systems were investigated in the presence of $\mathrm{LiCl}, \mathrm{NaCl}, \mathrm{KCl}$, and $\mathrm{CsCl}$. The diffuse layer potential $\psi_{\mathrm{dl}}$ is shown in the top row, and the regulation parameter $p$ in the middle 
row. In addition, the inner capacitance $C_{\text {in }}$ was calculated by from the two above parameters with Equations (8) and (9). These values are shown in the bottom row of Figure 4.

Let us first discuss the measured diffuse layer potentials, which are shown in the first row of Figure 4. All the potentials are negative and increase with increasing salt concentration. The same qualitative trend is predicted by the basic Stern model, albeit the predicted magnitudes of potentials are markedly larger, see Figure 1a. The experimental diffuse layer potentials are comparable for the silica and sulfate particles at $\mathrm{pH} 4$ and for both surfaces their values compare well with literature values extracted at the same conditions for similar particles $[38,54]$. At $\mathrm{pH} 10$, the silica particles show diffuse layer potentials that are substantially larger in magnitude than at $\mathrm{pH} 4$. This trend can be rationalized by the larger degree of ionization of the surface silanol groups.
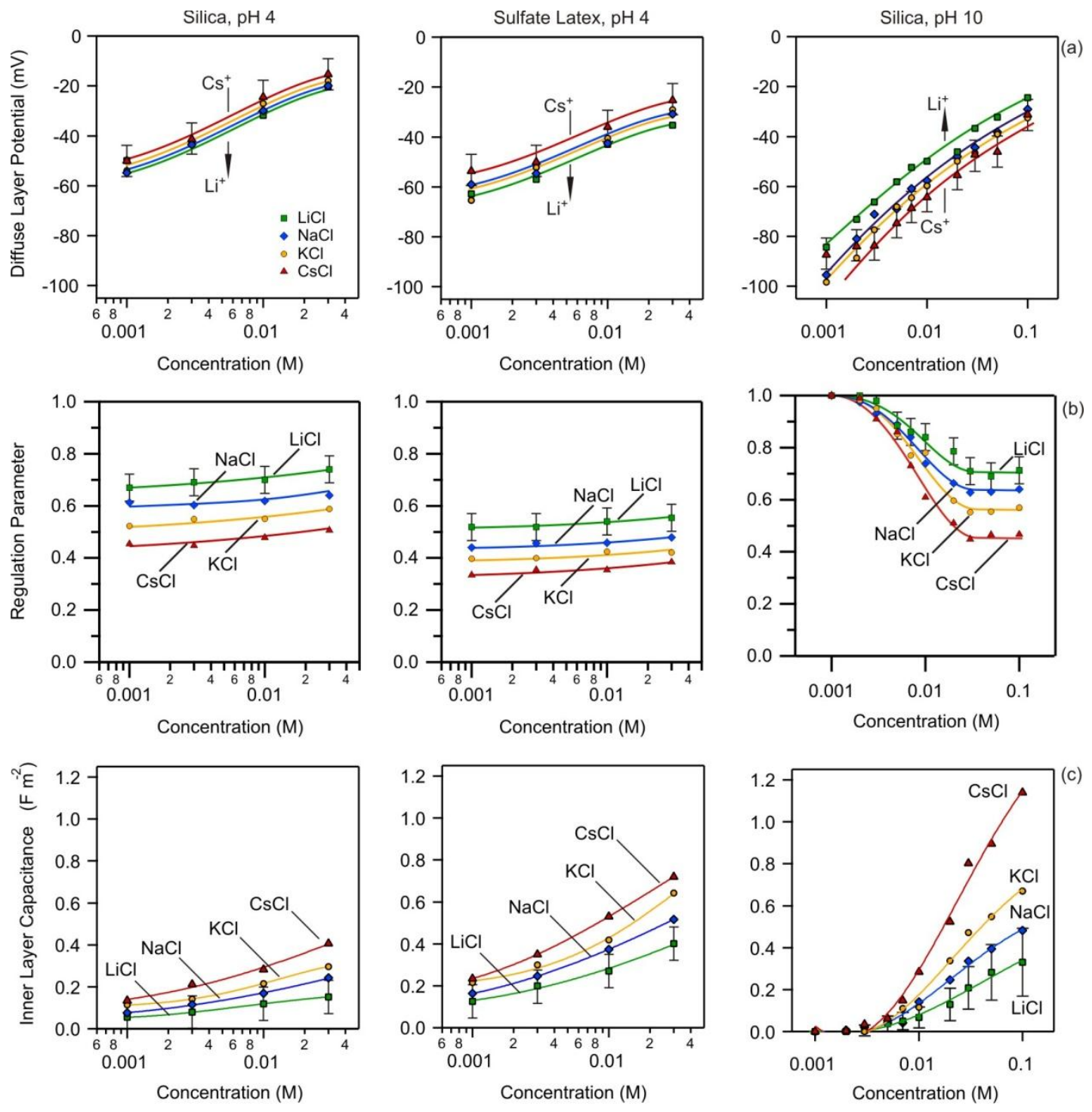

Figure 4. (a) Diffuse layer potentials, (b) regulation parameters, and (c) inner layer capacitances as a function of salt concentration extracted from the force measurements. The left column presents results for silica particles at $\mathrm{pH} 4$, the middle column for sulfate latex at $\mathrm{pH} 4$, and the right column shows the results for silica particles at $\mathrm{pH}$ 10. The measurements were done in $\mathrm{LiCl}, \mathrm{NaCl}, \mathrm{KCl}$, and $\mathrm{CsCl}$ aqueous solutions. Lines are only eye-guides. Error bars are only shown for one set of data but are comparable for all sets. 
Several ion specific effects can be further observed. At $\mathrm{pH} 4$, the potentials at a given concentration increase within the series $\mathrm{Li}^{+}, \mathrm{Na}^{+}, \mathrm{K}^{+}, \mathrm{Cs}^{+}$. This order is found for both silica and sulfate latex particles and it is consistent with the direct Hofmeister series $[40,53]$. This trend can be rationalized by the fact that the adsorption on a hydrophobic surface is stronger for weakly hydrated ions, like $\mathrm{Cs}^{+}$, than for strongly hydrated ions, like $\mathrm{Li}^{+}$. The stronger adsorption of $\mathrm{Cs}^{+}$leads to a higher diffuse layer potential than for $\mathrm{Li}^{+}$. These results are consistent with surface potentials measurements at $\mathrm{pH} 4$ in the same electrolytes with direct force measurements for silica [40] and by electrophoresis for sulfate latex particles [53]. For silica at $\mathrm{pH} 10$, the trend in diffuse layer potentials gets reversed, see the right panel of Figure 4a. Here the diffuse layer potentials decrease in the series $\mathrm{Li}^{+}, \mathrm{Na}^{+}$, $\mathrm{K}^{+}, \mathrm{Cs}^{+}$, which is also referred to as the inverse Hofmeister series. At high $\mathrm{pH}$, the silica surface becomes more hydrophilic due to the dissociation of the silanol groups, and now the strongly hydrated ions, like $\mathrm{Li}^{+}$, adsorb more strongly than the weakly hydrated ones, like $\mathrm{Cs}^{+}$. This inversion of the Hofmeister series for the silica surface by the solution $\mathrm{pH}$ was already inferred by direct force measurements by Sivan and coworkers [40]. The inverse Hofmeister series was also observed by means of potentiometric titration of silica particles [55,56]. However, electrokinetic potentials of silica nanoparticles at $\mathrm{pH} 10$ reported later $[57,58]$ are reversed with respect to the results presented here, and they follow the direct Hofmeister series. We suspect that this reversed trend is caused by the fact that the silica nanoparticles are less hydrophilic than the heated silica microparticles used in the present study. This difference is likely to be related to the hydrophobic to hydrophilic transition, which can explain the inversion of the Hofmeister series [59].

Let us now focus on the regulation parameters, which are shown in the second row of Figure 4. At $\mathrm{pH} 4$, the regulation parameter increases slightly with increasing concentration for the silica and sulfate latex surfaces. The values of regulation parameters for silica agree reasonably well with a constant value of $p=0.58$ in $\mathrm{KCl}$ at $\mathrm{pH} 4$ reported earlier [38]. An increase of regulation parameter with increasing salt concentration was also observed for sulfate latex particles for solution similar conditions [60], albeit the increase was reported to be stronger than the one found here. The increase of the regulation parameter with increasing salt concentration can be qualitatively rationalized with Equation (8), by assuming a constant inner capacitance and realizing that the diffuse layer capacitance increases with increasing salt concentration. However, this simple argument would predict a much stronger increase with the salt concentration that one actually observes. For silica at $\mathrm{pH} 10$, however, the regulation parameter decreases with increasing salt concentration. A similar decrease of regulation parameter with increasing salt concentration was also observed for silica at $\mathrm{pH} 10$ in the presence of multivalent co-ions [61], while a less pronounced decrease was observed for carboxylate latex particles at $\mathrm{pH} 3$ and 4 [60]. Ion specific effects can be observed again. The regulation parameter decrease in the series $\mathrm{Li}^{+}, \mathrm{Na}^{+}, \mathrm{K}^{+}, \mathrm{Cs}^{+}$. This trend is inversed as compared to the diffuse layer potentials.

The inner capacitance always increases with the salt concentration, see Figure 4c. At pH 4, this increase is moderate, while at $\mathrm{pH} 10$, this increase is very pronounced. Thus, an essential conclusion from these results is that the inner capacitance increases with the salt concentration. When this increase is weak, as for $\mathrm{pH} 4$, it compensates the increase of the diffuse layer capacitance. This interdependence leads to a regulation parameter that is approximately independent of the salt concentration, as observed experimentally. When this increase of the inner capacitance is more pronounced, as for silica at $\mathrm{pH} 10$, a decrease in the regulation parameter with increasing concentration is observed.

It is instructive to compare the experimental inner capacitance for silica at $\mathrm{pH} 10$ with the predictions from the basic Stern model shown in Figure 1b. One observes that the capacitances shown in Figure 1a, which were predicted with the parameters proposed by Hiemstra et al. [10], are too large, while measured capacitances much closer to the values predicted with the same model but using $C_{\mathrm{S}}=0.5 \mathrm{~F} / \mathrm{m}^{2}$, especially at lower salt concentrations. In accordance with the experiment, the basic Stern model also predicts an increase of the inner capacitance with salt concentration. However, the predicted increase is much weaker than the one observed. The explanations of this 
discrepancy could be that additional binding of other ions leads to a stronger increase of the binding capacitance, or that the Stern capacitance increases with increasing salt concentration.

The ion specific effects for the inner capacitances are again observed, and they consistently show a increase of this capacitance within the series $\mathrm{Li}^{+}, \mathrm{Na}^{+}, \mathrm{K}^{+}, \mathrm{Cs}^{+}$. This dependence is inversed with respect to the one observed in the regulation parameter and it follows the Hofmeister series.

The uncertainties in determination of the surface properties can be inferred from the error bars shown in Figure 4. Generally, the error bars are situated within the 10-20\% margin. However, the errors in the inner layer capacitance become large when the regulation parameter are close to unity, especially for silica at pH 10 and low salt concentrations. Further uncertainties could be related to the position plane of origin of the diffuse layer, which could be affected by the surface roughness, surface charge heterogeneities, and the presence of adsorbed ions at contact. These uncertainties are expected to be more pronounced at higher salt concentrations.

By fitting the measured force profiles, the diffuse layer potential $\psi_{\mathrm{dl}}$ and the regulation parameter $p$ can be found, and the inner capacitance $C_{\text {in }}$ can be calculated from these two parameters by using Equations (8) and (9). Within the basic Stern model, we have shown that the inner capacitance has to be interpreted as resulting from two capacitors in series, one being the Stern capacitance $C_{\mathrm{S}}$ and the other the binding capacitance $C_{\text {in }}$, see Equation (18). The calculations shown in Figure $1 \mathrm{~b}$ suggest that the binding capacitance is always much larger compared to the Stern capacitance at high salt concentrations. Therefore, the experimentally measured inner layer capacitance is dominated by the Stern capacitance at higher salt levels, while at lower salt levels contribution from the binding capacitance is present.

\subsection{Comparison with X-Ray Photoelectron Spectroscopy (XPS)}

Brown et al. [14,19] performed X-ray photoelectron spectroscopy measurements of silica particles in a liquid microjet. From these spectra they were able to determine the surface potential $\psi_{0}$ of the water-silica interface. With separate measurements of surface charge density by potentiometric titration and of diffuse layer potential by electrophoresis, they were able to extract the Stern capacitance. The Stern capacitance measured in this fashion is compared with our measurements of the inner capacitance in Figure 5. Since Brown et al. [14,19] exclusively focused on pH 10, we focus on these conditions only. Indeed, our measurements of the inner capacitance compare reasonably well with the ones obtained by XPS. Furthermore, they feature the same trend upon variation with the type of alkali metal ion and salt concentration. This agreement thus provides further support concerning the validity of our approach.
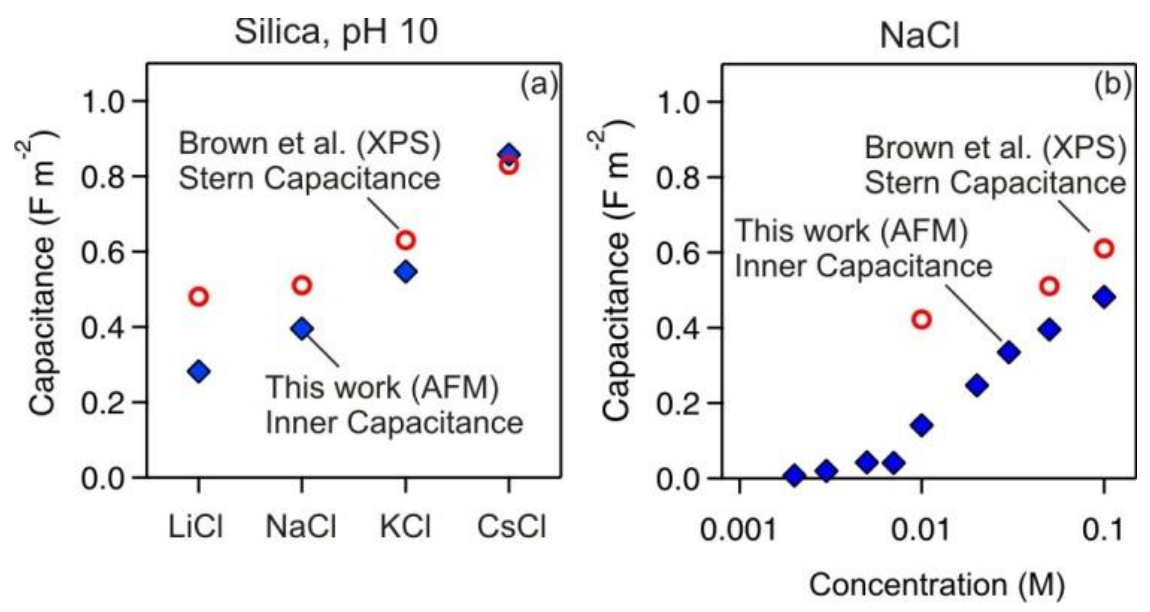

Figure 5. (a,b) Comparison to XPS data by Brown et al. [14,19] Capacitance as a function of cation type at $50 \mathrm{mM}$ salt concentration and $\mathrm{pH} \mathrm{10,} \mathrm{and} \mathrm{(b)} \mathrm{as} \mathrm{a} \mathrm{function} \mathrm{of} \mathrm{NaCl}$ concentration at $\mathrm{pH} 10$. Open symbols data by Brown et al., closed symbols this work. 
While the agreement between these two completely independent data sets is most encouraging, their interpretation of these findings is not straightforward. Brown et al. [14,19] have argued that the variation of the Stern layer capacitance is related to the thickness of the Stern layer $d_{\mathrm{S}}$, which can be inferred from the relation for a plane capacitor $[1,19]$ :

$$
C_{S}=\frac{\varepsilon_{S} \varepsilon_{0}}{d_{S}}
$$

where $\varepsilon_{S}$ is the relative dielectric constant within the capacitor. Brown et al. [14] interpreted the increase of the Stern capacitance in the series $\mathrm{Li}^{+}, \mathrm{Na}^{+}, \mathrm{K}^{+}, \mathrm{Cs}^{+}$by the variation of the Stern layer thickness, whereby they assumed a constant relative dielectric constant $\varepsilon_{S}=43$. [62] They argued that the Stern layer thickness corresponds to the apparent ionic diameter as extracted from limiting electrical conductivities by means of the Stokes law and, thus, is referred to as the Stokes diameter [63]. In our view, this interpretation is problematic, since the Stern layer thickness should be rather given by the ionic radius, and Stokes ion diameter does not represent the actual size of the ions, which can be measured more reliably by X-ray diffraction, neutron scattering, or X-ray absorption fine structure (XAFS) [64-66]. The deviations mainly occur due to neglect of dielectric friction and failure of the Stokes law at the molecular level $[67,68]$. Brown et al. [19] have further argued that the increase of the Stern capacitance with increasing salt concentration is caused by a decrease of the Stern layer thickness due to an increasing osmotic pressure of the electrolyte solution. We doubt that the modest osmotic pressure could induce such a massive compression of the Stern layer, which would be needed to explain the observed shift in the capacitance.

In our view, two alternative explanations of the observed dependencies are more likely. Molecular dynamic simulations suggest that the dielectric constant close to water-solid interfaces to differ substantially from their bulk values [57]. These differences are caused by non-uniformities in the concentration profile of the solvent and preferential orientation of the solvent molecules. These simulations suggest that the dielectric constant close to the interface increases with increasing salt concentration. This dependence is also consistent with recent spectroscopic data, which indicate that the water molecules are strongly oriented at low salt concentrations and more disordered at higher salt concentrations [15].

Another explanation could be that the increase of the inner capacitance with salt concentration is mainly caused by the contribution of the binding capacitance. Already the simple basic Stern model predicts such an increase, and specific adsorption of salt ions could enhance this contribution even further. Given the possibility of specific adsorption, the presence of such contributions in the measurements of the Stern capacitance Brown et al. [14,19] cannot be excluded either. The contribution of the binding capacitance could be also the reason for larger deviations between the capacitances measured by the two methods at lower salt concentrations.

\section{Conclusions}

Direct force measurements are used to extract properties of the inner layer near charged water-solid interfaces. The method relies on experimental force profiles measured between pairs of colloidal particles, and the inner capacitance is obtained by fitting the profiles with DLVO theory including charge regulation. The present results compare reasonably well with the recent measurements of the Stern capacitance on silica particles in a liquid microjet by XPS $[14,19]$. Given the completely independent nature of the techniques used, the good agreement between both sets of data provides strong evidence concerning the validity of the present approach.

The present results demonstrate that the inner layer capacitance increases in sequence $\mathrm{Li}^{+}, \mathrm{Na}^{+}$, $\mathrm{K}^{+}, \mathrm{Cs}^{+}$, which is consistent with the Hofmeister series. The inner capacitance further increases with increasing salt concentration. The variations of the capacitances with the ion type and salt concentration are probably related to the variation of dielectric constant in the inner layer and/or the variation of the binding capacitance. 
The advantage of the present technique is that properties of the inner layer can be studied for a wide range of systems, including the ones not accessible by other techniques. While XPS has been used to carry out similar measurements for silica [14,19], novel results for sulfate latex particles are presented here. One finds that the inner capacitance is larger than the one for silica, which could be related to the closer approach of the ions toward the more hydrophobic latex interface.

The present approach could be further extended to study particles of difference size, whereby nanoparticles can be mimicked with the AFM tip [34]. Flat substrates can also be investigated in the asymmetric setting. Especially for weakly charged surfaces, the asymmetric geometry allows to improve the accuracy of the extracted regulation parameters substantially $[37,48]$. For highly charged surfaces, on the other hand, the asymmetric and symmetric geometries lead to similar accuracies. Therefore, the latter geometry was chosen here.

Author Contributions: Conceptualization, A.S., P.M., M.B., and G.T.; Methodology, A.S., P.M., M.B., and G.T.; Software, P.M., M.B., and G.T.; Validation, A.S., P.M., M.B., and G.T.; Formal Analysis, A.S., P.M., M.B., and G.T.; Investigation, A.S., P.M., M.B., and G.T.; Resources, M.B.; Data Curation, A.S., P.M., M.B. and G.T.; Writing-Original Draft Preparation, M.B. and G.T.; Writing-Review \& Editing, A.S., P.M., M.B., and G.T.; Visualization, A.S., M.B. and G.T.; Supervision, M.B.; Project Administration, M.B. and G.T..; Funding Acquisition, M.B. and G.T.

Funding: This research was funded by Swiss National Science Foundation grant numbers 150631, 159874, and 162420 and the University of Geneva.

Conflicts of Interest: The authors declare no conflict of interest.

\section{References}

1. Russel, W.B.; Saville, D.A.; Schowalter, W.R. Colloidal Dispersions; Cambridge University Press: Cambridge, UK, 1989.

2. Elimelech, M.; Gregory, J.; Jia, X.; Williams, R.A. Particle Deposition and Aggregation: Measurement, Modeling, and Simulation; Butterworth-Heinemann Ltd.: Oxford, UK, 1995.

3. Bolto, B.; Gregory, J. Organic polyelectrolytes in water treatment. Water Res. 2007, 41, 2301-2324. [CrossRef] [PubMed]

4. Iselau, F.; Restorp, P.; Andersson, M.; Bordes, R. Role of the aggregation behavior of hydrophobic particles in paper surface hydrophobation. Colloid Surf. A 2015, 483, 264-270. [CrossRef]

5. Zanini, M.; Hsu, C.P.; Magrini, T.; Marini, E.; Isa, L. Fabrication of rough colloids by heteroaggregation. Colloid Surf. A 2017, 532, 116-124. [CrossRef]

6. Squires, T.M.; Quake, S.R. Microfluidics: Fluid physics at the nanoliter scale. Rev. Mod. Phys. 2005, 77, 977-1026. [CrossRef]

7. Bard, A.J.; Faulkner, L.R. Electrochemical Methods: Fundamentals and Applications; John Wiley: New York, NY, USA, 2001.

8. Naji, A.; Kanduc, M.; Forsman, J.; Podgornik, R. Perspective: Coulomb fluids - weak coupling, strong coupling, in between and beyond. J. Chem. Phys. 2013, 139, 150901. [CrossRef] [PubMed]

9. Stern, O. Zur Theorie der elektrolytischen Doppelschicht. Z. Elektrochem. 1924, 30, 508-516.

10. Hiemstra, T.; de Wit, J.C.M.; van Riemsdijk, W.H. Multisite proton adsorption modeling at the solid-solution interface of (hydr)oxides: A new approach 2. Application to various important (hydr)oxides. J. Colloid Interface Sci. 1989, 133, 105-117. [CrossRef]

11. Machesky, M.L.; Wesolowski, D.J.; Palmer, D.A.; Ichiro-Hayashi, K. Potentiometric titrations of rutile suspensions to 250 degrees C. J. Colloid Interface Sci. 1998, 200, 298-309. [CrossRef]

12. Kobayashi, M.; Skarba, M.; Galletto, P.; Cakara, D.; Borkovec, M. Effects of heat treatment on the aggregation and charging of Stöber-type silica. J. Colloid Interface Sci. 2005, 292, 139-147. [CrossRef] [PubMed]

13. Dove, P.M.; Craven, C.M. Surface charge density on silica in alkali and alkaline earth chloride electrolyte solutions. Geochim. Cosmochim. Acta 2005, 69, 4963-4970. [CrossRef]

14. Brown, M.A.; Abbas, Z.; Kleibert, A.; Green, R.G.; Goel, A.; May, S.; Squires, T.M. Determination of surface potential and electrical double-layer structure at the aqueous electrolyte-nanoparticle interface. Phys. Rev. X 2016, 6. [CrossRef] 
15. Boamah, M.D.; Ohno, P.E.; Geiger, F.M.; Eisenthal, K.B. Relative permittivity in the electrical double layer from nonlinear optics. J. Chem. Phys. 2018, 148, 222808. [CrossRef] [PubMed]

16. Darlington, A.M.; Jarisz, T.A.; DeWalt-Kerian, E.L.; Roy, S.; Kim, S.; Azam, M.S.; Hore, D.K.; Gibbs, J.M. Separating the $\mathrm{pH}$-dependent behavior of water in the Stern and diffuse layers with varying salt concentration. J. Phys. Chem. C 2017, 121, 20229-20241. [CrossRef]

17. Myalitsin, A.; Urashirna, S.H.; Nihonyanagi, S.; Yamaguchi, S.; Tahara, T. Water structure at the buried silica/aqueous interface studied by heterodyne-detected vibrational sum-frequency generation. J. Phys. Chem. C 2016, 120, 9357-9363. [CrossRef]

18. Bourg, I.C.; Lee, S.S.; Fenter, P.; Tournassat, C. Stern layer structure and energetics at mica-water interfaces. J. Phys. Chem. C 2017, 121, 9402-9412. [CrossRef]

19. Brown, M.A.; Goel, A.; Abbas, Z. Effect of electrolyte concentration on the Stern layer thickness at a charged interface. Angew. Chem. Int. Ed. 2016, 55, 3790-3794. [CrossRef] [PubMed]

20. Perkin, S.; Kampf, N.; Klein, J. Long-range attraction between charge-mosaic surfaces across water. Phys. Rev. Lett. 2006, 96, 038301. [CrossRef] [PubMed]

21. Israelachvili, J.; Min, Y.; Akbulut, M.; Alig, A.; Carver, G.; Greene, W.; Kristiansen, K.; Meyer, E.; Pesika, N.; Rosenberg, K.; et al. Recent advances in the surface forces apparatus (SFA) technique. Rep. Prog. Phys. 2010, 73, 036601. [CrossRef]

22. Helfricht, N.; Mark, A.; Dorwling-Carter, L.; Zambelli, T.; Papastavrou, G. Extending the limits of direct force measurements: Colloidal probes from sub-micron particles. Nanoscale 2017, 9, 9491-9501. [CrossRef] [PubMed]

23. Gutsche, C.; Keyser, U.F.; Kegler, K.; Kremer, F. Forces between single pairs of charged colloids in aqueous salt solutions. Phys. Rev. E 2007, 76, 031403. [CrossRef] [PubMed]

24. Crocker, J.C.; Grier, D.G. Microscopic measurement of the pair interaction potential of charge-stabilized colloid. Phys. Rev. Lett. 1994, 73, 352-355. [CrossRef] [PubMed]

25. Derjaguin, B.; Landau, L.D. Theory of the stability of strongly charged lyophobic sols and of the adhesion of strongly charged particles in solutions of electrolytes. Acta Phys. Chim. 1941, 14, 633-662. [CrossRef]

26. Verwey, E.J.W.; Overbeek, J.T.G. Theory of Stability of Lyophobic Colloids; Elsevier: Amsterdam, The Netherlands, 1948.

27. Labbez, C.; Jonsson, B.; Pochard, I.; Nonat, A.; Cabane, B. Surface charge density and electrokinetic potential of highly charged minerals: Experiments and Monte Carlo simulations on calcium silicate hydrate. J. Phys. Chem. B 2006, 110, 9219-9230. [CrossRef] [PubMed]

28. Kanduc, M.; Naji, A.; Forsman, J.; Podgornik, R. Dressed counterions: Strong electrostatic coupling in the presence of salt. J. Chem. Phys. 2010, 132, 124701. [CrossRef] [PubMed]

29. Hartley, P.G.; Larson, I.; Scales, P.J. Electrokinetic and direct force measurements between silica and mica surfaces in dilute electrolyte solutions. Langmuir 1997, 13, 2207-2214. [CrossRef]

30. Dishon, M.; Zohar, O.; Sivan, U. From repulsion to attraction and back to repulsion: The effect of $\mathrm{NaCl}, \mathrm{KCl}$, and $\mathrm{CsCl}$ on the force between silica surfaces in aqueous solution. Langmuir 2009, 25, 2831-2836. [CrossRef] [PubMed]

31. Ninham, B.W.; Parsegian, V.A. Electrostatic potential between surfaces bearing ionizable groups in ionic equilibrium with physiologic saline solutions. J. Theor. Biol. 1971, 31, 405-408. [CrossRef]

32. Carnie, S.L.; Chan, D.Y.C. Interaction free energy between plates with charge regulation: A linearized model. J. Colloid Interface Sci. 1993, 161, 260-264. [CrossRef]

33. Behrens, S.H.; Borkovec, M. Electrostatic interaction of colloidal surfaces with variable charge. J. Phys. Chem. B 1999, 103, 2918-2928. [CrossRef]

34. Zhao, C.; Ebeling, D.; Siretanu, I.; van den Ende, D.; Mugele, F. Extracting local surface charges and charge regulation behavior from atomic force microscopy measurements at heterogeneous solid-electrolyte interfaces. Nanoscale 2015, 7, 16298-16311. [CrossRef] [PubMed]

35. Trefalt, G.; Behrens, S.H.; Borkovec, M. Charge regulation in the electrical double layer: Ion adsorption and surface interactions. Langmuir 2016, 32, 380-400. [CrossRef] [PubMed]

36. Borkovec, M.; Behrens, S.H. Electrostatic double layer forces in the case of extreme charge regulation. J. Phys. Chem. B 2008, 112, 10795-10799. [CrossRef] [PubMed] 
37. Popa, I.; Sinha, P.; Finessi, M.; Maroni, P.; Papastavrou, G.; Borkovec, M. Importance of charge regulation in attractive double-layer forces between dissimilar surfaces. Phys. Rev. Lett. 2010, 104, 228301. [CrossRef] [PubMed]

38. Valmacco, V.; Elzbieciak-Wodka, M.; Herman, D.; Trefalt, G.; Maroni, P.; Borkovec, M. Forces between silica particles in the presence of multivalent cations. J. Colloid Interf. Sci. 2016, 472, 108-115. [CrossRef] [PubMed]

39. Sader, J.E.; Larson, I.; Mulvaney, P.; White, L.R. Method for the calibration of atomic force microscope cantilevers. Rev. Sci. Instrum. 1995, 66, 3789-3798. [CrossRef]

40. Morag, J.; Dishon, M.; Sivan, U. The governing role of surface hydration in ion specific adsorption to silica: An AFM-based account of the Hofmeister universality and its reversal. Langmuir 2013, 29, 6317-6322. [CrossRef] [PubMed]

41. Trefalt, G.; Palberg, T.; Borkovec, M. Forces between colloidal particles in aqueous solutions containing monovalent and multivalent ions. Curr. Opin. Colloid Interf. Sci. 2017, 27, 9-17. [CrossRef]

42. Ducker, W.A.; Senden, T.J.; Pashley, R.M. Direct measurement of colloidal forces using an atomic force microscope. Nature 1991, 353, 239-241. [CrossRef]

43. Valle-Delgado, J.J.; Molina-Bolivar, J.A.; Galisteo-Gonzalez, F.; Galvez-Ruiz, M.J.; Feiler, A.; Rutland, M.W. Hydration forces between silica surfaces: Experimental data and predictions from different theories. J. Chem. Phys. 2005, 123, 034708. [CrossRef] [PubMed]

44. Grabbe, A.; Horn, R.G. Double layer and hydration forces measured between silica sheets subjected to various surface treatments. J. Colloid Interface Sci. 1993, 157, 375-383. [CrossRef]

45. Acuna, S.M.; Toledo, P.G. Nanoscale repulsive forces between mica and silica surfaces in aqueous solutions. J. Colloid Interface Sci. 2011, 361, 397-399. [CrossRef] [PubMed]

46. Adler, J.J.; Rabinovich, Y.I.; Moudgil, B.M. Origins of the non-DLVO force between glass surfaces in aqueous solution. J. Colloid Interface Sci. 2001, 237, 249-258. [CrossRef] [PubMed]

47. Montes Ruiz-Cabello, F.J.; Trefalt, G.; Oncsik, T.; Szilagyi, I.; Maroni, P.; Borkovec, M. Interaction forces and aggregation rates of colloidal latex particles in the presence of monovalent counterions. J. Phys. Chem. $B$ 2015, 119, 8184-8193. [CrossRef] [PubMed]

48. Moazzami-Gudarzi, M.; Trefalt, G.; Szilagyi, I.; Maroni, P.; Borkovec, M. Nanometer-ranged attraction induced by multivalent ions between similar and dissimilar surfaces probed by the atomic force microscope (AFM). Phys. Chem. Chem. Phys. 2016, 18, 8739-8751. [CrossRef] [PubMed]

49. Valmacco, V.; Elzbieciak-Wodka, M.; Besnard, C.; Maroni, P.; Trefalt, G.; Borkovec, M. Dispersion forces acting between silica particles across water: Influence of nanoscale roughness. Nanoscale Horiz. 2016, 1, 325-330. [CrossRef]

50. Ackler, H.D.; French, R.H.; Chiang, Y.M. Comparisons of Hamaker constants for ceramic systems with intervening vacuum or water: From force laws and physical properties. J. Colloid Interface Sci. 1996, 179, 460-469. [CrossRef]

51. Bevan, M.A.; Prieve, D.C. Direct measurement of retarded van der Waals attraction. Langmuir 1999, 15, 7925-7936. [CrossRef]

52. Elzbieciak-Wodka, M.; Popescu, M.; Montes Ruiz-Cabello, F.J.; Trefalt, G.; Maroni, P.; Borkovec, M. Measurements of dispersion forces between colloidal latex particles with the atomic force microscope and comparison with Lifshitz theory. J. Chem. Phys. 2014, 140, 104906. [CrossRef] [PubMed]

53. Oncsik, T.; Trefalt, G.; Borkovec, M.; Szilagyi, I. Specific ion effects on particle aggregation induced by monovalent salts within the Hofmeister series. Langmuir 2015, 31, 3799-3807. [CrossRef] [PubMed]

54. Montes Ruiz-Cabello, F.J.; Trefalt, G.; Maroni, P.; Borkovec, M. Accurate predictions of forces in the presence of multivalent ions by Poisson-Boltzmann theory. Langmuir 2014, 30, 4551-4555. [CrossRef] [PubMed]

55. Tadros, T.F.; Lyklema, J. Adsorption of potential determining ions at silica-aqueous electrolyte interface and role of some cations. J. Electroanal. Chem. 1968, 17, 267-275. [CrossRef]

56. Abendroth, R.P. Behavior of a pyrogenic silica in simple electrolytes. J. Colloid Interface Sci. 1970, 34, 591-596. [CrossRef]

57. Bonthuis, D.J.; Gekle, S.; Netz, R.R. Profile of the static permittivity tensor of water at interfaces: Consequences for capacitance hydration interaction and ion adsorption. Langmuir 2012, 28, 7679-7694. [CrossRef] [PubMed] 
58. Redondo, A.B.; Jordan, I.; Ziazadeh, I.; Kleibert, A.; Giorgi, J.B.; Worner, H.J.; May, S.; Abbas, Z.; Brown, M.A. Nanoparticle-induced charge redistribution of the air-water interface. J. Phys. Chem. C 2015, 119, 2661-2668. [CrossRef]

59. Schwierz, N.; Horinek, D.; Netz, R.R. Anionic and cationic Hofmeister effects on hydrophobic and hydrophilic surfaces. Langmuir 2013, 29, 2602-2614. [CrossRef] [PubMed]

60. Montes Ruiz-Cabello, F.J.; Trefalt, G.; Maroni, P.; Borkovec, M. Electric double layer potentials and surface regulation properties measured by colloidal probe atomic force microcopy. Phys. Rev. E 2014, 90, 012301. [CrossRef] [PubMed]

61. Uzelac, B.; Valmacco, V.; Trefalt, G. Interactions between silica particles in the presence of multivalent coions. Soft Matter 2017, 13, 5741-5748. [CrossRef] [PubMed]

62. Sverjensky, D.A. Prediction of surface charge on oxides in salt solutions: Revisions for 1:1 (M+L-) electrolytes. Geochim. Cosmochim. Acta 2005, 69, 225-257. [CrossRef]

63. Kielland, J. Individual Activity Coefficients of Ions in Aqueous Solutions. J. Am. Chem. Soc. 1937, 59, 1675-1678. [CrossRef]

64. Marcus, Y. Ionic radii in aqueous solutions. Chem. Rev. 1988, 88, 1475-1498. [CrossRef]

65. Persson, I. Hydrated metal ions in aqueous solution: How regular are their structures? Pure Appl. Chem. 2010, 82, 1901. [CrossRef]

66. Kameda, Y.; Uemura, O. Neutron Diffraction Study on the Structure of Highly Concentrated Aqueous $\mathrm{LiBr}$ Solutions. Bull. Chem. Soc. Jpn. 1993, 66, 384-389. [CrossRef]

67. Kay, R.L. The current state of our understanding of ionic mobilities. Pure Appl. Chem. 1991, $63,1393$. [CrossRef]

68. Marcus, Y. Are Ionic Stokes Radii of Any Use? J. Solut. Chem. 2012, 41, 2082-2090. [CrossRef]

(C) 2018 by the authors. Licensee MDPI, Basel, Switzerland. This article is an open access article distributed under the terms and conditions of the Creative Commons Attribution (CC BY) license (http:/ / creativecommons.org/licenses/by/4.0/). 